\title{
Conventional vs. Liquid Based Cytology in Fine Needle Aspirates of Lung
} and Mediastinal Masses

\author{
Garima Singh $^{1 *}$, Preeti Agarwal' ${ }^{2}$, Madhu Mati Goel${ }^{2}$, Madhu Kumar ${ }^{2}$ and Durg Pratap Singh ${ }^{2}$
}

${ }^{1}$ G.C.R.G Institute of Medical Science and Hospital, Lucknow, Uttar Pradesh, India

${ }^{2}$ King George's Medical University, Shah Mina Road, Chowk, Lucknow, Uttar Pradesh, India

\begin{abstract}
Background: Lung cancer most common worldwide cancer, early diagnosis makes effective treatment in lung cancer. Liquid Based Cytology (LBC) for non-gynae specimens has gained popularity over conventional Cytology (CS) during last one decade because of increased sensitivity and specificity.
\end{abstract}

Aim and objectives: To assess the diagnostic utility of LBC versus CS in fine needle aspirates of lung and mediastinal mass lesions. Compare cyto-morphological parameters in terms of adequacy, interpretation, concordance and diagnostic efficiency.

Materials and Methods: Eighty -two cases suspected of lung or mediastinal mass lesions underwent imagine guided Transbronchial Needle aspiration (TBNA) or Trans Thoracic Needle aspiration (TTFNA). Fine needle passes were made for both conventional and LBC smears. All smears were examined and diagnosis was correlated in each case with histopathology as gold standard for final diagnosis. Results: Histopathology of 82 cases, 21 were non -neoplastic and 61 were malignant respectively. 34 cases of adenocarcinoma, 15 cases of squamous cell carcinoma, 5 cases of adenosquamous cell carcinoma, 4 cases of small cell carcinoma, 1 cases of poorly differentiated 1 case of carcinoid and 1 cases of germ cell tumor. LBC were diagnosed 17 non -neoplastic and 54 were malignant respectively. 25 cases of adenocarcinoma, 11 cases of squamous cell carcinoma, 5 cases of adenosquamous cell carcinoma and 3 cases of small cell carcinoma, CS was diagnosed 4 non -neoplastic and 37 were malignant respectively. 24 cases of adenocarcinoma, 7 cases of squamous cell carcinoma, 4 cases of adenosquamous cell carcinoma and 2 cases of small cell carcinoma.

Conclusion: Concordance of LBC with histopathology was found in higher proportion (72\%) as compared to conventional smear $(48.8 \%)(p=0.004)$, because percentage of adequacy for LBC $(85.37 \%)$ was higher as compared to CS $(56.1 \%)(\mathrm{P}<0.001)$. The assessment of all the morphological parameters were predominantly equal in both methods except in few cases.

Keywords: Conventional; Histopathology; Lung cancer; Surepath; Cytology

\section{Introduction}

Lung cancer is most common worldwide cancer [1]. Even though there is recent and marked advancements in molecular understanding of lung cancer and targeted therapies, the annual incidence of death rate high due to late presentation of clinical sign and symptoms [2]. Hence, early diagnosis is very instrumental in management.

Fine Needle aspiration (FNA) cytology is well established as an alternate to open biopsies, for making definitive diagnosis, even though histopathological examination (HPE) remains the gold standard. FNA has proved to be quick, effective, physically non-traumatic, non-invasive and inexpensive method, which may provide equivalent results, if correlated with clinical history and CT or MRI scan to localize the mass lesions. There are two methods of smear preparation after aspiration i.e., Conventional Smearing (CS) and Liquid Based Cytology (LBC) [3].

Liquid Based Cytology (LBC) for non-gynae specimens has gained popularity over conventional smear (CS) during last one decade especially in developed countries; however in developing nations CS is still popular.

Lung masses are usually evaluated by endoscopic biopsies and cytology. In present study we evaluated the diagnostic utility of aspiration cytology of lung and mediastinal mass lesions between the two methods of smear preparation (LBC vs CS). To establish the diagnostic ease and efficiency we compared cyto-morphological parameters in terms of adequacy, interpretation and concordance.

\section{Material and Methods}

A total of 102 cases were registered at our center for evaluation of lung and mediastinal aspirates during 2014-2015 (Endobronchial Ultrasound Trans Bronchial Needle Aspiration (EBUS-TBNA) and CT guided Transthoracic Fine Needle Aspiration (TTFNA)). Since biopsy sample was not available in 20 cases, hence 82 out of 102 cases were included in the study. 39 of them were EBUS-TBNA and 43 were TTFNA samples.

All the lesions were aspirated by a chest physician, at least 2-3 passes, adequate sample were collected and conventional smears were made, two fixed in 95\% alcohol for Haemtoxylin and Eosin ( $\mathrm{H}$ and $\mathrm{E}$ ) staining. Fresh material by a separate prick was rinsed in LBC preservative (CytoRich-RED BD ${ }^{\mathrm{TM}}$-Atlanta, USA). No split samples were taken. Samples were taken to cytology laboratory for further processing.

The conventional and LBC smears were examined separately for cytological features. Cyto-histological correlation was made.

${ }^{*}$ Corresponding author: Garima Singh, G.C.R.G Institute of Medical Science and Hospital, Lucknow, Uttar Pradesh, India, Tel: 8960822079; E-mail: garisingh007@gmail.com

Received January 09, 2017; Accepted April 04, 2017; Published April 07, 2017

Citation: Singh G, Agarwal P, Goel MM, Kumar M, Singh DP (2017) Conventional vs. Liquid Based Cytology in Fine Needle Aspirates of Lung and Mediastinal Masses. J Pulm Respir Med 7: 400. doi: 10.4172/2161-105X.1000400

Copyright: ( 2017 Singh G, et al. This is an open-access article distributed under the terms of the Creative Commons Attribution License, which permits unrestricted use, distribution, and reproduction in any medium, provided the original author and source are credited. 
Histopathology was considered as gold standard for final diagnosis in all cases, specifically where the results of cytology were equivocal.

\section{Results}

Of the histopatholgically diagnosed cases; benign were $25.6 \%$ while malignant cases were $74.4 \%$. Among malignant cases, Non-Small Cell Lung Cancer (NSCLC (91.8\%)) was more frequent histological type as compared to Small Cell Lung Cancer (SCLC (6.5\%)) (Figures 1 and 2). Among NSCLC Adenocarcinoma (Figure 1a LBC and b CS) was most frequent followed by Squamous cell carcinoma (Figure 1c LBC and d CS).

Among 82 cases included in the present study the inadequacy rates were significantly lower for conventional smears as compared to $\mathrm{LBC}$ as shown in Table 1 . When diagnostic efficacy of the smearing technique was compared to final tissue diagnosis LBC $(80.9 \%)$ was significantly better in terms of identification of benign lesions as compared to CS (19\%). Moreover the results in malignant diagnosis was also better but were no significant as seen in Table 2 .

Cytomorphologic features like cellularity, chromatin nucleoli were also evaluated in the present study. For statistical analysis of these variables in diagnosis, we normalized our observations by making following assumptions a) inadequate samples were excluded from the study (Table 1) and b) cyto-morphological features which were not seen or were not applicable in benign and malignant smears were counted as 1 (i.e. $\mathrm{LBC}=\mathrm{CS}$ ) ( i.e., 5 cases of comparable adequacy). Hence the assessment cyto-morphological features of forty-one cases were predominantly equal as compared to conventional smear. However better preservation of nucleoli, chromatin texture, and cellular pleomorphism was found in LBC as comparison to conventional smear Table 3.

Overall accuracy of LBC with histological diagnosis was significantly higher than conventional (59/82; $72 \%$ vs $40 / 82$; $48.8 \%)$. Hence our results suggest that LBC is no doubt a better method of smear preparation from cytological material obtained from lung aspirates (Table 4).

\section{Discussion}

Use of LBC for non- gynae specimens as well has increased for the past decade [4]. Authors have studied the application of Thin Layer Cytology in exfoliative and aspiration cytology of pulmonary, urinary, gastrointestinal, endocrine, breast and salivary gland specimen and in the diagnosis of serous effusion. They found that ThinPrep was better than conventional $[5,6]$ in samples from all the above sites they analysed.

Food and Drug Administration (FDA) approved liquid based cytopreparatory techniques are ThinPrep (TP) method and SurePath (SP). ThinPrep (TP) method is based on filtration method and SurePath (SP) method based on centrifugation methods. Specimens

\section{LBC $\quad$ CS}

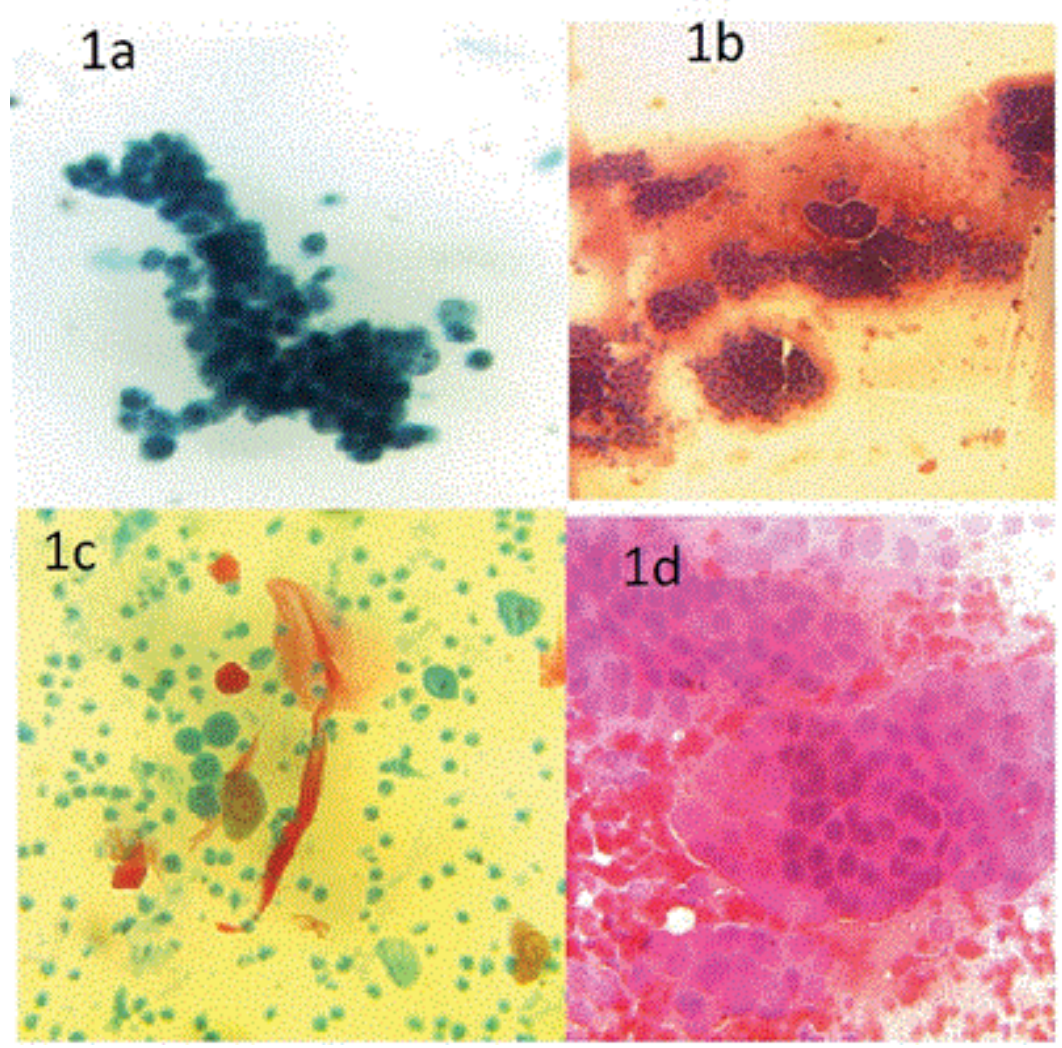

Figure 1: a: LBC smear of Adenocarcinoma at X200, showing vesicular chromatin with prominent nucleoli. b: Conventional smear of Adenocarcinoma at X200, showing cells arrangement in acini pattern. c: LBC smear of Squamous cell carcinoma at X200, showing few atypical squamous cell. d: Conventional smear of Squamous cell carcinoma at X200. 
for LBC technique are collected in preservative fluids provided by the manufacturer in both the techniques; for example CytoRich vials in SurePath $\mathrm{BD}^{\mathrm{TM}}$ system. CytoRich preservatives are of two types based on their compositions. CytoRich Blue is an alchol-based fixative including ethanol and methanol. CytoRich Red is a formalin based fixative including isopropanol, ethylene glycol, methanol, and formalin which is recommended for collection of hemorrhagic samples by the manufacturer.

NSCLC was $91.8 \%$ of all diagnosed cancers in the present study. The above observation is quite close to the data of Molina et al. [7] who also observed NSCLC to be $85 \%$ of all lung cancer in their study. In NSCLC, adenocarcinoma was highest 34/56 (60.7\%). Results were concordance with results of various workers in India have reported prevalence of lung cancer [8].

Adequacy rates of aspiration samples reported in literature ranges from $63-97 \%$ for conventional methods [9-12]. The total adequacy rates in lung masses of the present study were significantly lower as compared to the literature. Aspiration sample of lung masses is an invasive procedure done either under bronchoscopic or CT (Computed tomography) guidance. The expertise of the physician who is taking out the sample is very instrumental. One of the most important reasons of recommending use of LBC in aspiration samples of lung masses in our study is lower inadequacy rate; adequacy of samples determined by LBC $(85.37 \%)$ was significantly higher than conventional methods (56.10\%). This observation is because LBC (SurePath $\mathrm{BD}^{\mathrm{TM}}$ ) uses automated technique to concentrate the cellular yield by standardized

\begin{tabular}{|l|c|c|c|c|}
\hline \multirow{2}{*}{} & \multicolumn{2}{|c|}{ Liquid Based Cytology } & \multicolumn{2}{c|}{ Conventional Cytology } \\
\cline { 2 - 5 } & No. & $\%$ & No. & $\%$ \\
\hline Adequate & 70 & 85.37 & 46 & 56.1 \\
\hline Inadequate & 12 & 14.63 & 36 & 43.9 \\
\hline Total & 82 & 100 & 82 & 100 \\
\hline
\end{tabular}

Table 1: Comparison of adequacy in conventional cytology and liquid based cytology. $x 2=16.966 ; p<0.001$. procedure. Moreover the washing of the aspiration needle in collection fluid ensures complete transfer of cellular material. In CS preparation part of material is retained in needle hub.

To improve the adequacy of CS onsite evaluation of conventional smears by using ultrafast Papnicolau stain has been recommended [13]. Preparation of LBC smears at the site of FNA is practically not possible. Inadequate cases $9.8 \%$ in CS while $8.8 \%$ in LBC were excluded for calculation of the concordance between LBC and CS in term of cellularity, morphology of cells, efficacy and ease of interpretation of diagnosis.

As far as cyto-morphological parameters were concerned among adequate samples both the smear preparation techniques were equally good for evaluation of preserved cells. However better nucleoli preservation (19.5\% in LBP and $9.7 \%$ in CS), chromatin clumping (26.8\% in LBP and $24.4 \%$ in CS) and pleomorphism (17\% in LBC and $14.6 \%$ in CS.) were observed in LBC in comparison to CS. This perhaps was because of immediate delivery of material in the preservative fluid and the smears prepared by automate techniques leading to better visualization of nuclear details. These were concordant with studies of various authors where they found almost equal sensitivity and specificity of both techniques in interpretation of aspiration samples of TTFNA from peripheral nodular lesions of lung [14].

LBC techniques have been evaluated in past in lung samples which constituted mainly sputum, bronchial brushings, BAL $[15,16]$. Most of the authors have found LBC preparations better to CS in assessment of the above samples from lung masses. However, few with varying results have evaluated TBNA and TTFNA. Various authors observed better sensitivity and specificity of TBNA samples by using LBC [17]. They found that sensitivity of LBC was $82.1 \%$ while in CS it was $56 \%$. The specificity of LBC was $87.5 \%$, and for CS it was $82.5 \%$.

In our study, diagnosis by LBC was found to be concordant with the histology diagnosis in majority of cases, $80.95 \%$ benign cases and $72.13 \%$ malignant cases.

\begin{tabular}{|c|c|c|c|c|c|c|c|}
\hline \multirow[t]{2}{*}{ Histological Diagnosis } & \multirow{2}{*}{$\begin{array}{c}\text { Total number of } \\
\text { cases diagnosed } \\
\text { by HPE }\end{array}$} & \multicolumn{2}{|c|}{$\begin{array}{l}\text { No. Of cases } \\
\text { diagnosed by } \\
\text { Conventional }\end{array}$} & \multicolumn{2}{|c|}{$\begin{array}{c}\text { No. Of cases } \\
\text { diagnosed by LBC }\end{array}$} & \multicolumn{2}{|c|}{ Statistical significance } \\
\hline & & No. & $\%$ & No. & $\%$ & $x^{2}$ & $\mathbf{P}$ \\
\hline Benign & 21 & 4 & 19.05 & 17 & 80.95 & 16.095 & $<0.001$ \\
\hline Adeno carcinoma & 34 & 24 & 70.59 & 25 & 73.53 & 0.073 & 0.787 \\
\hline Squamous cell carcinoma & 15 & 7 & 46.67 & 11 & 73.33 & 2.222 & 0.136 \\
\hline Adeno squamous carcinoma & 5 & 4 & 80 & 5 & 100 & 1.111 & 0.292 \\
\hline Small cell carcinoma & 4 & 2 & 50 & 3 & 75 & 0.533 & 0.465 \\
\hline $\begin{array}{l}\text { Other carcinoma (carcinoid, germ cell tumor, poorly differentiated } \\
\text { malignancy) }\end{array}$ & 3 & 0 & 0 & 0 & 0 & 0 & 1 \\
\hline
\end{tabular}

Table 2: Comparative diagnostic Efficacy of cytological techniques.

\begin{tabular}{|c|c|c|c|c|c|c|c|}
\hline \multirow{2}{*}{ Cytomorpho-logical parameters } & \multirow{2}{*}{ Total } & \multicolumn{2}{|c|}{ Conventional Equivalent to LBC } & \multicolumn{2}{|c|}{ LBC better than conventional } & \multicolumn{2}{|c|}{ Conventional better than LBC } \\
\hline & & No. & $\%$ & No. & $\%$ & No. & $\%$ \\
\hline Cellularity & 41 & 21 & 51.22 & 11 & 26.83 & 9 & 21.95 \\
\hline Pleomorphism & 41 & 28 & 68.29 & 7 & 17.07 & 6 & 14.63 \\
\hline Chromatin clumping & 41 & 20 & 48.78 & 11 & 26.83 & 10 & 24.39 \\
\hline Nucleoli & 41 & 29 & 70.73 & 8 & 19.51 & 4 & 9.76 \\
\hline Efficacy & 41 & 22 & 53.66 & 9 & 21.95 & 10 & 24.39 \\
\hline Ease of Interpretation & 41 & 21 & 51.22 & 10 & 24.39 & 10 & 24.39 \\
\hline
\end{tabular}

Table 3: Cytomorphological details of parameters observed in liquid based and conventional cytology. 
We observed statistically significant difference in concordance between LBC and CS with respect to histology, $(p=0.004)$. LBC showed a statistically significant increase in sensitivity as compared to conventional methods.

Few authors in their study of LBC on TTFNA samples showed LBC had inferior yield of diagnosis as comparison to CS method [18]. Their observation was in contrast to other observations by others and our data, as far as lung aspirates were concerned. This could be due to smaller sample size of their study only 30 samples were analysed. The material obtained from 2-3 passes was used to make CS as compared to only single pass was used to collect LBC sample. So differential sample were a major drawback of their study [18].

Moreover there is always a learning curve in LBC interpretation and training is needed before someone starts interpreting these preparations because of fine alterations of morphology and cellular size. In the present study, conventional smear was found to be inadequate in $9.8 \%$ while in LBC inadequacy rate was $8.8 \%$ of cases but no false positive diagnosis was made in any of the case, thus specificity of both techniques beings $100 \%$ for diagnosis of malignancy.

An interesting observation was found when we evaluated the results in terms of histological diagnosis of NSCLC. Diagnostic concordance with histology was better by LBC in Squamous cell carcinoma $(73.33 \%$ by LBC versus $46.67 \%$ by conventional method) and comparable for

\begin{tabular}{|l|c|c|c|c|}
\hline \multirow{2}{*}{ Histological Diagnosis } & \multicolumn{2}{|c|}{ Conventional } & \multicolumn{2}{|c|}{ LBC } \\
\cline { 2 - 5 } & No. & $\%$ & No. & $\%$ \\
\hline Concordance & 40 & 48.8 & 59 & 72 \\
\hline No concordance & 34 & 41.5 & 15 & 18.3 \\
\hline Could not be commented & 8 & 9.8 & 8 & 8.8 \\
\hline
\end{tabular}

Table 4: Concordance of conventional and liquid based cytology with histology diagnosis. $\mathrm{X} 2=11.014(\mathrm{df}=2) ; \mathrm{p}=0.004$ (Chi-square test) (Significant). techniques as far as adenocarcinoma was concerned $(73.3 \%$ by LBC and $70.6 \%$ by $\mathrm{CS})$.

In our study, we observed some advantages in LBC over CS. It was less time consuming as per interpretation with smaller screening area $(13 \mathrm{~mm})$, clear background. Our study supports the view of other studies who found that TP preparations are superior to $\mathrm{CP}$ with regard to clear background, monolayer cell preparation and cell preservation. Also, it is easier and less time consuming to screen and interpret TP preparation because the cells are limited to smaller areas on clear background with excellent cellular preservation. However, TP preparations are more expensive than $\mathrm{CP}$ and require some experience for interpretation.

LBC offers an advantage of cell- block preparation from left over material for further ancillary techniques but procedures like cytogenetic and molecular cannot be performed which requires unfixed cellular material.

Every technique has some advantages and disadvantages. LBC also has some disadvantages, which are loss of background during processing. Though some hint of it remains but majority is lost.

Morphologically we found that smearing and molding were less pronounced in LBC smears in small cell carcinoma lung and some amount of cellular elongation was observed in these cases [19]. The above observation is due to suspension of cells in liquid media. However, dispersed population, monomorphic nuclei and chromatin clumping gave a clue to diagnosis (Figure 2).

\section{Conclusion}

We recommend use of liquid based preparations in lung aspirates i.e., TTFNA and EUS-TBNA as they are obtained while either the patient undergoes CT or endoscopy due to better adequacy and ease of interpretation. Moreover the overall diagnostic accuracy is also significantly higher as compared to conventional smearing; thus a

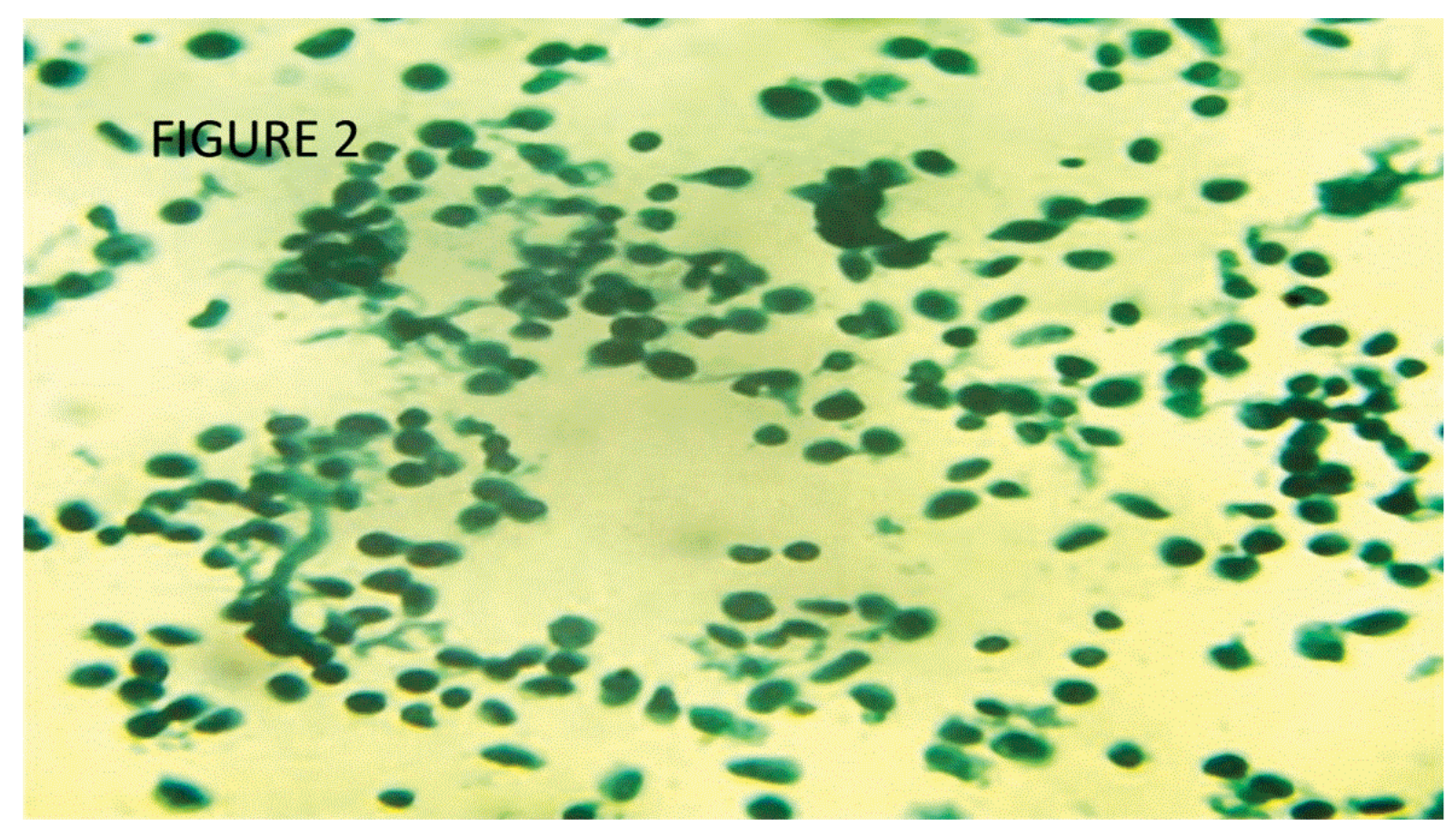

Figure 2: LBC smear of Small cell carcinoma at X200, showing uniform small cells, with scant cytoplasm in clear background. 
Citation: Singh G, Agarwal P, Goel MM, Kumar M, Singh DP (2017) Conventional vs. Liquid Based Cytology in Fine Needle Aspirates of Lung and Mediastinal Masses. J Pulm Respir Med 7: 400. doi: 10.4172/2161-105X.1000400

cellular smear with better-preserved morphology obtained from LBC becomes furthermore obligatory.

\section{References}

1. Ferlay J, Soerjomataram I, Ervik M, Dikshit R, Eser S, et al. (2013) GLOBOCAN 2012 v1.0. Cancer Incidence and Mortality Worldwide: IARC CancerBase No. 11. Lyon, France: International Agency for Research on Cancer.

2. Bowen EF, Rayner CF (2002) Patient and GP led delays in the recognition of symptoms suggestive of lung cancer. Lung Cancer 37: 227-228.

3. Kobayashi $\mathrm{Y}$, Uehara T, Ota $\mathrm{H}$ (2011) Liquid based thinlayer cytology can be routinely used in samples obtained via fiberoptic bronchoscope. Acta Cytol 55: 69-78.

4. Hoda RS (2007) Non-gynecologic cytology on liquid-based preparations: A morphologic review of facts and artifacts. Diagn Cytopathol 35: 621-634.

5. Parfitt JR, McLachlin CM, Weir MM (2007) Comparison of ThinPrep and conventional smears in salivary gland fine-needle aspiration biopsies. Cancer 111: 123-129.

6. Joseph L, Edwards JM, Nicholson CM, Pitt MA, Howat AJ (2002) An audit of the accuracy of fine needle aspiration using a liquid-based cytology system in the setting of a rapid access breast clinic. Cytopathology 13:343-349.

7. Molina JR, Yang P, Cassivi SD, Schild SE, Adjei AA (2008) Non-small cell lung cancer: epidemiology, risk factors, treatment, and survivorship. Mayo Clin Proc 83: 584-594.

8. Behera D, Balamugesh T (2004) Lung cancer in India. Indian J Chest Dis Allied Sci 46: 269-281.

9. Stewart CJ, Stewart IS (1996) Immediate assessment of fine needle aspiration cytology of lung. J Clin Pathol 49: 839-843.

10. Tian Q, Chen LA, Wang HS, Zhu BH, Tian L, et al. (2010) Endobronchial ultrasound-guided transbronchial needle aspiration of undiagnosed mediastinal lymphadenopathy. Chin Med J 123: 2211-2214.

11. Khazai L, Kundu UR, Jacob B, Patel S, Sneige N, et al. (2011) Endobronchial ultrasound-guided transbronchial needle aspiration biopsy is useful evaluating mediastinal lymphadenopathy in a cancer center. Cytojournal 8: 10.

12. Yadav RK, Sen R, Agarwal S, Aggarwal S (2010) CT-guided FNAC of intrathoracic mass lesions--a study among 35 patients. J Indian Med Assoc 108: $571-574$.

13. Bando K, Haba R, Kushida Y, Kadota K, Katsuki N, et al. (2011) Utility of immediate cytologic diagnosis of lung masses using ultrafast Papanicolaou stain. Lung Cancer 72: 172-176.

14. Imura J, Abe K, Uchida Y, Shibata M, Tsunematsu K, et al. (2014) Introduction and utility of liquid-based cytology on aspiration biopsy of peripheral nodular lesions of the lung. Oncol Lett 7: 669-673.

15. Astall E, Atkinson C, Morton N, Goddard MJ (2003) The evaluation of liquidbased "CytoSED" cytology of bronchioalveolar lavage specimens in the diagnosis of pulmonary neoplasia against conventional direct smears. Cytopathology 14: 143-149.

16. Yang Y, Pan QJ, Teng MF, Li ZL, Zhao LL, et al. (2008) Application of protein markers in combination with ThinPrep bronchial brush cytology in classification of lung cancer subtypes. Zhonghua zhong liu za zhi 30: 616-619

17. Qui T, Zhu K, Lai M, Shi J, Wang K (2015) Liquid-based cytology preparation can improve cytological assessment of endobronchialultrasound guided transbronchial needle aspiration. Act Cytologica 59: 139-143.

18. Mendea M, Leeuwena A, Bakkerb M, Koegelenberg C (2012) Liquid based vs conventional cytology for evaluation of fine needle aspiration biopsies obtained by pulmonary physicians A pilot study. Erasmus Journal of Medicine 3: 1.

19. Kim S, Owens CL (2009) Analysis of ThinPrep cytology in establishing the diagnosis of small cell carcinoma of lung. Cancer 117: 51-56. 